\title{
An Enhanced Cluster Gateway Switch Routing Protocol (ECGSR) for Congestion Control using AODV Algorithm in MANET
}

\author{
K. Devarajan \\ Assistant Professor \\ Department of Electrical Engg. \\ FEAT, Annamalai University \\ Annamalai Nagar-608002 \\ Chidambaram, Tamil Nadu, India
}

\author{
V. Padmathilagam, PhD \\ Assistant Professor \\ Department of Electrical Engg. \\ FEAT, Annamalai University \\ Annamalai Nagar-608002 \\ Chidambaram, Tamil Nadu, India
}

\begin{abstract}
In Mobile Ad-hoc networks, without any fixed access point the mobile nodes are formed randomly. The congestion may increase while the network traffic increases due to the dynamic topology of nodes and limited capacity of memory. In Ad-hoc networks, the congestion is an important issue because it will lead to loss of packet, long delay and overhead of packets and so the performance of Ad-hoc networks also reduces. The congestion control is based on controlling the traffic in which too many packets exist in the part of the subnet. This manuscript proposes the Enhanced Clusterhead Gateway Switch Routing Protocol (ECGSR) with the basis of Ad-hoc On-demand Distance Vector Routing (AODV) based mechanism for congestion control. In this process, the cluster head plays the main role in monitoring the congestion in Adhoc networks, by reducing the number of transmissions and by creating the routes on need. This proposed scheme is to reduce the loss of packets, routing overhead and end-to-end delay during the data packet transmission. So it accomplishes the throughput, better packet delivery ratio, low end-to-end delay and overhead by reducing the congestion in Ad-hoc networks using the chosen ECGSR protocol based on AODV basis. The ECGSR is well-organized to control the congestion than the Clusterhead Gateway Switch Routing Protocol (CGSR) with the basis of Destination Sequence Distance Vector (DSDV).
\end{abstract}

\section{Keywords}

ECGSR (Enhanced Clusterhead Gateway Switch Routing Protocol), Congestion control, MANET (Mobile Ad-hoc Networks), Cluster head, AODV (Ad-hoc On-demand Distance Vector Routing), DSDV (Destination Sequence Distance Vector), TCL (Tool Command Language).

\section{INTRODUCTION}

A MANET (Mobile Ad-hoc Networks) is a type of Ad-hoc networks that configures itself continuously and can change locations. As MANETS use wireless connections to connect the different networks, are portable and infrastructure-less networks. So each device can move independently in any direction and its links can be changed frequently to the other devices [1]. The MANET characteristics are

- Wireless communication networks

- Infrastructure less networks

- No centralized controller

- Dynamic Network topology
- Inner reciprocal trust

- Frequently routing updates

- Mobile nodes execute the roles of hosts and routers

Some applications of MANETS are [1]

- Distress aid operations

- Development of defense

- Imperative business meetings

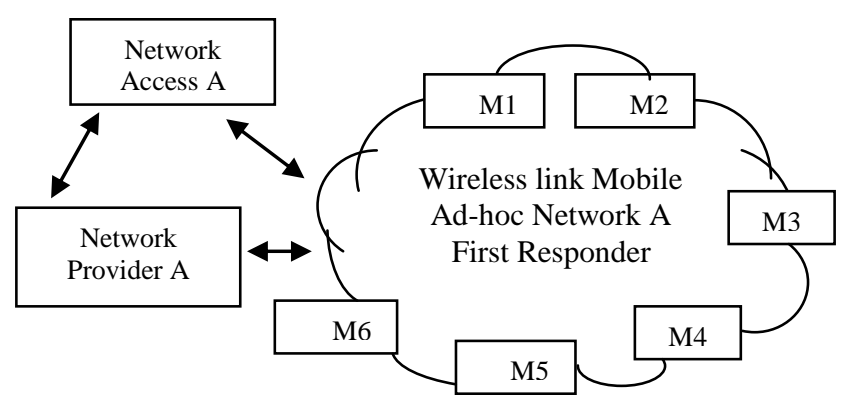

Fig.1 Basic Structure of MANET

Each device in Mobile Ad-hoc Networks must transmit traffic, unconnected to its personal use and hence there must be routed to sustain traffic in the transmission of packets. The heavy traffic creates a congestion that can lead to losses of the packet, reduce the energy of networks and increase the overhead [2]. So congestion control is needed to avoid packet losses, to improve the energy and reduce the overhead in the MANET. The congestion occurs if the number of packets sent is more than the network capacity that the number of packets it can handle.

In a MANET, the routing protocols are used in managing the traffic and congestion also can be reduced. The routing protocols are partitioned into proactive, reactive and hybrid protocols [3] that are based on the methods of table driven. In this paper, the Enhanced Clusterhead Gateway Switch Routing Protocol (ECGSR) which consists of AODV based mechanism to overcome the congestion in an efficient manner is developed and the performance is compared to other routing protocols.

\section{RELATED WORK}

Vinay. P. Viradia [1], proposed a new protocol Enhanced AODV (E-AODV) which is a modified version of AODV with enhanced packet delivery ratio and minimized end- to- 
end delay.

Neelam Phate and Madhvi Saxena et al. [2] worked on an enhancement to the AODV routing protocol that consists of a cluster-based mechanism for supporting congestion control in MANET which offers a QoS alert path. The clustering and the selection of the cluster-head is the main feature of this approach on the basis of the congestion status of the nodes. This protocol is very competent in dealing congestion by accomplishing QoS constraints with energy efficiency.

Subburam S et al. [3] focused on predictive congestion control routing protocol for wireless Ad-hoc networks called as PCCAODV. Unlike traditional AODV, predictive congestion index of a node is calculated as the ratio of current queue occupancy over total queue size at the node level. PCCAODV uses the upstream nodes and downstream nodes of a congested node and initiates route finding process $\mathrm{Bi}$ directionally to find an alternate non congested path between them for transmitting data based on a congestion index. Suppose that the process finds more non congested multi paths and decides a finer single path for transmitting data.

Heesook Choi and William Enck [4] proposed an Anonymous and Secure Reporting of Traffic Forwarding Activity in Mobile Ad-hoc Networks. The proposed protocol uses nodes on the data path to securely create reports of packet forwarding. Nodes of reporting are selected arbitrarily and furtively so that malicious nodes cannot modify their behavior based upon the monitoring position. The authenticity and integrity of reports are conserved through the use of secure link layer acknowledgments and monitoring reports. By forwarding the report to multiple destinations (source and destination), the robustness of the reporting mechanism are strengthened.

Hemant Kumar et al. [5] use the effective TCP Congestion Control AODV routing, which consists of congestion monitoring of the rate control and queue length basis. The total congestion status is calculated in congestion monitoring. They proposed congestion control in the particular channel; rate of packet failure, packet line length and traffic rate based total congestion, average and ratio of packet dropping to monitor the congestion status in route establishment. On the networks, based on the congestion ordinary, the congestion a smaller amount based routing is established to reduce the failure of packet, long delay, high overhead.

Labiod $\mathrm{H}$ et al. [6] focused on a novel routing protocol named QoS Adaptive Source Routing Protocol (QoS-ASR). Through the aggregate flows, it is an adaptive Soft-QoS protocol. QoSASR uses the mechanism of source routing defined to avoid channel overhead and to develop scalability by the Dynamic Source Routing (DSR) unicast protocol. It operates QoS criteria, taking into account application requirements, combined with networks state associated enforcements. This scheme is evaluated via simulations and is compared to DSR.

Baisakh et al. [7] proposed an Energy Efficient Dynamic Source Routing (EEDSR) protocol algorithm that is chosen a node as a source node and then selects a set of node as destination node. The best path is selected and then the parameters are collected for each source and destination pair. Above all the source and destination pair the average value of the parameters is taken. Between the number of nodes and the average parameter value this method is frequented for all the networks of different dimension and a graph was planned. EEDSR algorithm not only enlarges the network's life, but also minimizes the effort required during the route maintenance period. EEDSR prohibits back overflowing of the packets and thus reduces the network congestion.

Rahim Rashidi et al. [8] worked on the congestion control section that guarantees the stability of networks and of the most highly trust nodes the distribution of the load is completed. By the agents the recommended model is executed on the network nodes. To show the presented model prospect, it is completed on the AODV protocol. In look up on to the executed simulation of the OPNET environment using the presented model improves the efficiency of the networks, although trust is malicious nodes and congestion on the networks.

Senthilkumaran. T, et al. [9] propose a method for dynamic congestion detection and control routing (DCDR) in Ad-hoc networks based on the estimations of the average queue length at the node level. A node discovers the present congestion level and sends a warning message to its neighbors using the average queue length. The neighbors then attempt to locate a congestion-free alternative path to the destination. In Ad-hoc networks, this dynamic congestion estimate mechanism supporting congestion control ensures reliable communication within the MANET.

Govardhan Reddy Kamatam et al. [10] discusses the Self Congestion Prediction Algorithm for congestion prediction and modified AODV with SCP and proposes a novel A-SCP protocol for efficient routing and congestion to reduce network overhead and manage the throughput of the transmission without failures in the networks. To assess the proposal they simulated in different random mobility and scalable network environment which shows an improvisation in packet delivery and decline in control overhead and end-toend delay.

Veerlapati Raju et al. [11] proposed a Capacity-Optimized Cooperative (COCO) topology management theme to enhance the network capability in MANETs by together considering each higher layer network capability and physical layer cooperative communications. Through simulations, it shows that physical layer cooperative communications have important impacts on the network capability, and also the topology management theme will well improve the network capability in MANETs with cooperative communications.

\section{METHODOLOGY}

\subsection{Enhanced Clusterhead Gateway Switch Routing Protocol (ECGSR)}

Enhanced Clusterhead Gateway Switch Routing Protocol (ECGSR) uses the basis of ADOV (Ad-hoc On-demand Distance Vector Routing) algorithm.

In a MANET, the mobile nodes are gathered to form the cluster and the cluster head is selected based on the energy level of mobile nodes that are in cluster head communication range belonging to its clusters. The gateway node is a node that connects two or more cluster heads. Due to frequent selection of cluster head, the cluster scheme can cause the reduction performance in dynamic networks. Therefore, the ECGSR uses the Least Cluster Change (LCC) algorithm. The change of cluster head occurs only if a change in networks causes two clusters-heads to come into one cluster or one of the nodes move out of the range of all the cluster-heads in LCC.

In general, the packet source conveys the packet to its cluster head, the packet can be send to the gateway, mobile node that links this cluster head and nearest next cluster head along the 
route to the destination from this cluster head. The gateway node conveys it to that cluster head and so on until the end cluster head is achieved in this route. Finally, the cluster head sends the packet to the destination.

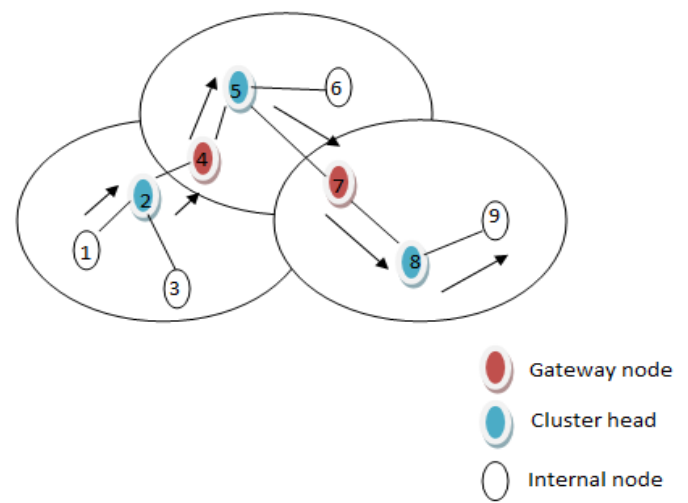

Fig.2 Example of CGSR routing from node 1 to node 9

Fig. 2 shows an example of CGSR routing from node 1 to node 9. This figure has three cluster heads and two gateway nodes. Each node maintains tree structures of cluster member that is designed from each node to its relative cluster head. Each node transmits its cluster member based on their sequence number to assure the new routes and updates its trees after receiving other node mapping and transmits using the algorithm of AODV. In addition, each node maintains tree structures of cluster member that describes the next hop to achieve the cluster of destination.

A node finds the nearest cluster-head along the route to the destination according to the cluster member trees and the routing tree on receiving a packet. Then it consults its routing table to find the next hop in order to reach the cluster-head selected in step one and transmits the packet to that node. If the source moves, then it can initiate route discovery to the destination cluster head. If one of the intermediate nodes moves to the moved node's neighbor realizes the link failure and sends a link failure notification to its upstream neighbors and so on till it reaches the source upon which the source can reinitiate route discovery if needed.

\subsection{Selection of cluster head}

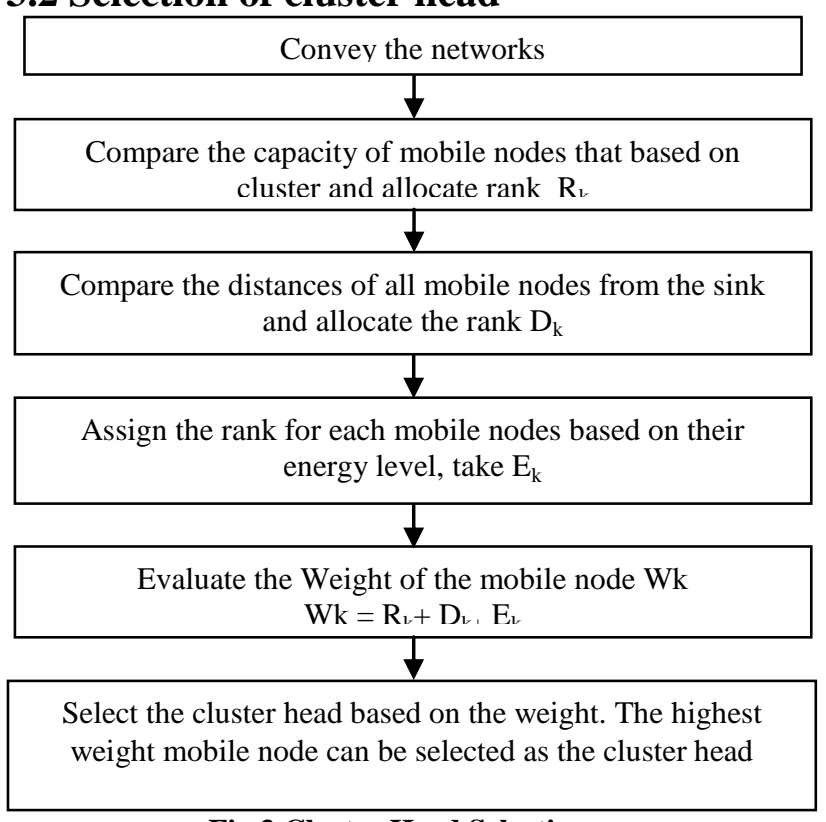

Fig.3 Cluster Head Selection
In this process, each cluster head can maintain two tales that are

i. Table for routing: This table manages the route information for each transmission of packets.

ii. Table for cluster member: It maintains the information on its each cluster member.

\subsection{AODV Mechanism}

In this paper, an Enhanced Clusterhead Gateway Switch Routing Protocol (ECGSR) is proposed using the AODV routing protocol.

For Ad-hoc mobile networks, the Ad-hoc On Demand Distance Vector (AODV) routing algorithm is a routing protocol mapped. AODV is able to routs both as unicast and multicast. It based on on-demand algorithm; by the source nodes, it builds the routes between the nodes that are desired. By the sources, AODV asserts these routes as long as they are needed. In addition, AODV create trees which join group members of multicast. In AODV, the trees are formed by the group members and the nodes needed to connect the members. It uses sequence numbers to assure the new routes. AODV does not have a loop and it has self-starting and scales to huge numbers of mobile nodes.

The source transmits a route request packet to find a path to the destination. Till it achieves the destination node the neighbors in turn sends the packet to their neighbors until it achieves an intermediate node that has new route information about the destination. If it has already arrived, a node rejects a route request packet. The packet of route request uses sequence numbers to assure that the routes are loop free and to make sure that if the intermediate nodes reply to requests of the route they respond with the latest information only.

It also records in its tables the node from which the first copy of the request came when a node forwards a route request packet to its nearest nodes. For the route reply packet, this report is used to build the reverse path. It uses only concurrent connections, because the route reply packet follows the reverse path of a packet of the route request. Along the path the nodes enter the forward route into their tables when the route reply packet traverses back to the source.

If the source moves, then it can reinitiate route discovery to the destination node. While one of the intermediate nodes moves, then they moved nodes neighbor realizes the link failure and sends a link failure notification to its upstream neighbors and so on till it reaches the source upon which the source can reinitiate route discovery if needed.

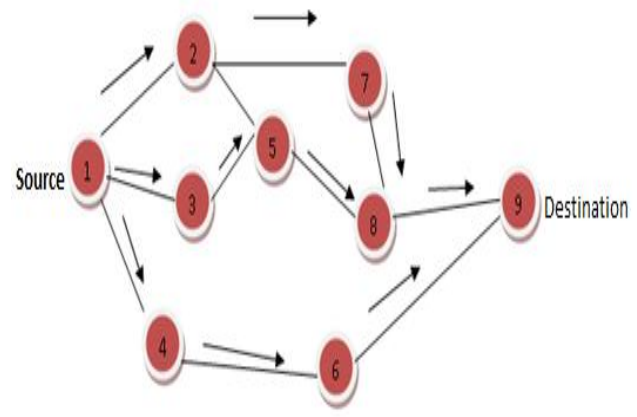

Fig.4 Route Request Packet Propagation

The above Fig.4 shows that the route request packet propagation in nine numbers of mobile nodes. The source sends the route request to available all routes to reach the 
destination for packet transmission. The route request propagation is used in Ad-hoc On Demand mechanism to find the best route to transmit the packet from source mobile node to destination mobile node.

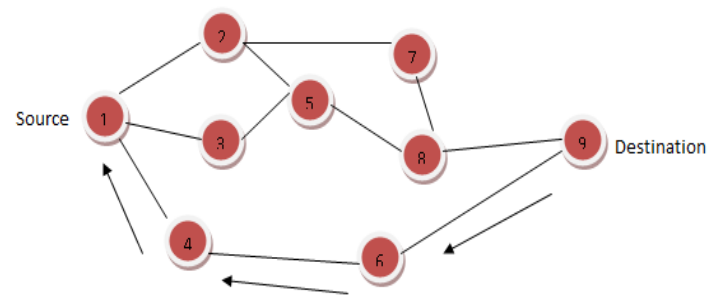

Fig.5 Path taken by the Route Reply Packet

The Fig. 5 shows that the path taken by the route reply packet. In this figure the shortest path is taken by the route reply packet. The selected path is best for transmission of packet delivery compared to other routes.

\section{RESULTS AND DISCUSSIONS}

The proposed ECGSR protocol is simulated using the Networks Simulator 2 (NS2) and it is contrasted to existing CGSR routing protocol. Table.1 shows the network parameters for the simulation. The performance of the proposed ECGSR protocol on AODV basis is presented and compared with the existing CGSR on DSDV basis. The performance of both protocols can be compared in terms of end-to-end delay, routing overhead, ratio of packet delivery and average of throughput analysis.

In NS-2, the frontend of the program is written in TCL (Tool Command Language). The backend of the NS-2 simulator is written in $\mathrm{C}++$ and when the TCL program is compiled, a trace file and Networks Animator (NAM) file are created which define the movement pattern of the nodes and keeps track of the number of packets sent, number of hops between 2 nodes, connection type etc. at each instance of time.

The Performance of protocols is evaluated by using the below parameter values:

Table.1 Simulation Parameters

\begin{tabular}{|l|c|}
\hline \multicolumn{1}{|c|}{ Parameter name } & Parameter values \\
\hline Number of nodes & $100,150,200,250$ \\
\hline Simulation area & $1000 \mathrm{~m}^{2}$ \\
\hline Time of simulation & $175 \mathrm{~ms}$ \\
\hline Protocols name & CGSR and ECGSR \\
\hline Size of Packet & 5000 \\
\hline Packet rate & $250 \mathrm{~kb}$ \\
\hline Initial energy & $100 \mathrm{~J}$ \\
\hline
\end{tabular}

\subsection{End-to-End delay analysis}

In the destination of networks, the average time taken by a data packet to reach is the end-to-end delay. By the process route discovery and queue in the transmission of a data packet, the delay can be occurred. The successfully delivered data packets are only counted. The end-to-end delay is calculated by the following formula:

$\sum$ (arrive time - send time) / $\sum$ (Number of connections)

If protocol has the lower end-to-end delay value, then the protocol has the better performance in the Mobile Ad-hoc Networks.
Table.2 End-to-End delay of protocols

\begin{tabular}{|c|c|c|}
\hline $\begin{array}{c}\text { Number of } \\
\text { nodes }\end{array}$ & CGSR & ECGSR \\
\hline 100 & 30.589 & 27.225 \\
\hline 150 & 36.964 & 32.869 \\
\hline 200 & 47.367 & 44.745 \\
\hline 250 & 52.967 & 48.997 \\
\hline
\end{tabular}

The above table shows the values of end-to-end delay in ECGSR and CGSR protocols. From the end-to-end delay analysis, an Enhanced Cluster Gateway Switch Routing Protocol has the lower values of end-to-end delay compared to existing CGSR for all chosen number of nodes. The ECGSR protocol reduces the end-to-end delay in the data packet transmission.

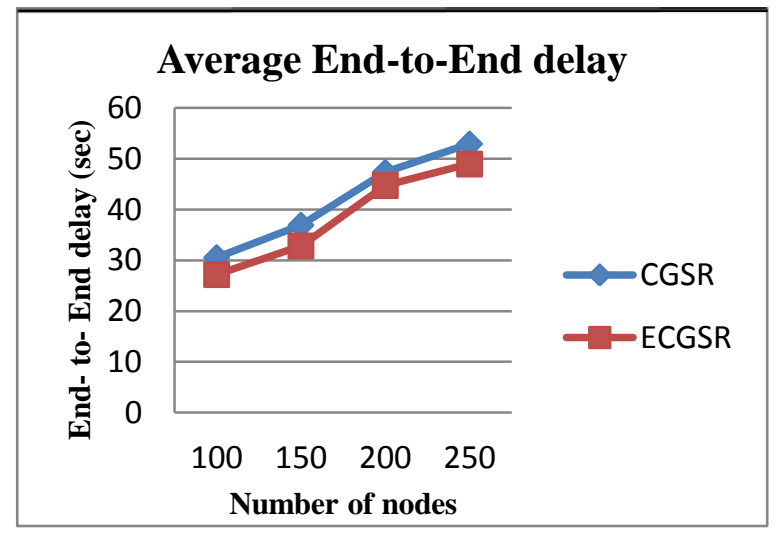

Fig.6 Average of End-to-End delay

The above Fig.6 shows that the simulation results of average of end-to-end delay of proposed ECGSR and existing CGSR protocol.

\subsection{Overhead of Routing Analysis}

Within networks, nodes often change their location. So, some routes are generated in the routing table which leads to unnecessary routing overhead. The routing overhead can be calculated by the following equation:

$R O=P F+S N+T R$

Where, RO stands for Routing Overhead, PF refers to the number of packets failed to reach the destination, SN stands for the messages that are having the serial numbers, while TR indicates the trigger messages.

Table.3 Routing Overhead of Protocols

\begin{tabular}{|c|c|c|}
\hline $\begin{array}{l}\text { Number of } \\
\text { nodes }\end{array}$ & CGSR & ECGSR \\
\hline 100 & 8.425 & 8.257 \\
\hline 150 & 8.369 & 8.136 \\
\hline 200 & 8.628 & 8.454 \\
\hline 250 & 8.599 & 8.352 \\
\hline
\end{tabular}

The above Table. 3 shows the routing overhead of ECGSR and CGSR. In the MANET, if protocol has the lower routing overhead, then it can provide better presentation. In the above table the ECGSR has the lower routing overhead compared to existing CGSR, consequently the ECGSR can give the best routing for packet transmission because this protocol can manage the routing overhead and take routing decisions effect. 


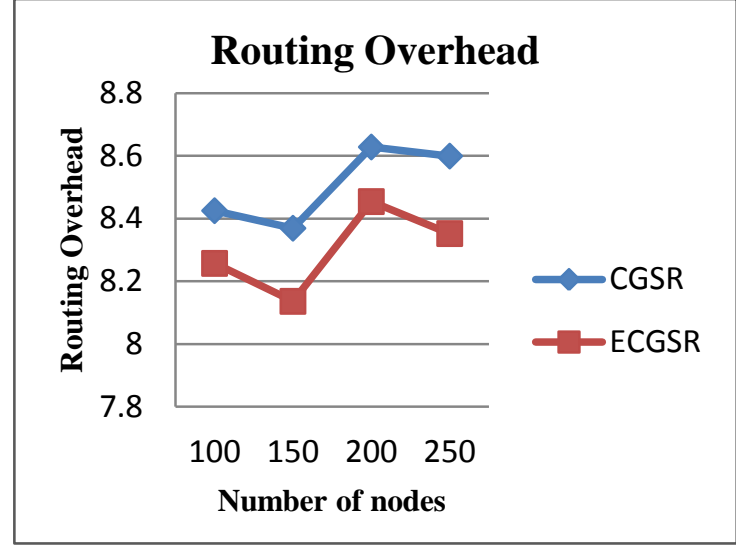

Fig.7 Routing Overhead

The above Fig.7 Shows simulation graph the routing overhead of CGSR and proposed ECGSR protocol.

\subsection{Packet Delivery Ratio Analysis}

The packet delivery ratio can be calculated by the ratio of the number of data packets delivered from source to destination node. It describes the delivered data levels to the destination mobile node. The ratio of packet delivery is measured by following equation.

$\sum$ Number of packets received / $\sum$ Number of packets sent

Table.4 Packet Delivery Ratio values of protocols

\begin{tabular}{|c|c|c|}
\hline $\begin{array}{c}\text { Number of } \\
\text { nodes }\end{array}$ & $\begin{array}{c}\text { CGSR } \\
\text { \% }\end{array}$ & $\begin{array}{c}\text { ECGSR } \\
\text { \% }\end{array}$ \\
\hline 100 & 91.02 & 95 \\
\hline 150 & 93.85 & 97.23 \\
\hline 200 & 92.23 & 95.51 \\
\hline 250 & 95.52 & 99.12 \\
\hline
\end{tabular}

The above Table. 4 shows that packet ratio value of protocols. From our analysis of packet delivery percentage, the ECGSR has a high packet delivery ratio compared to the existing protocol CGSR. While protocol has the high packet delivery ratio then the protocol has the best presentation. The protocol ECGSR can reduce the packet loss during the data packet broadcast; hence it will give the better packet delivery ratio. The proposed protocol ECGSR provides the best delivery ratio in the Mobile Ad-hoc Networks compared to existing protocol CGSR.

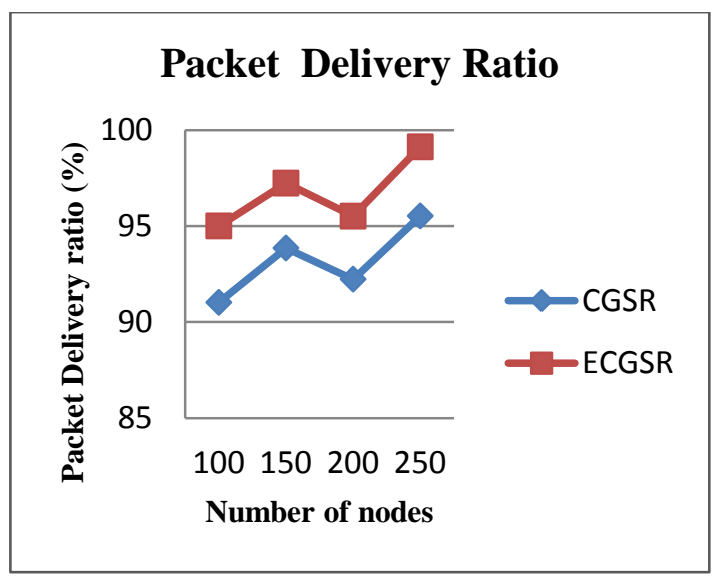

Fig.8 Packet Delivery Ratio

The Fig. 8 shows the packet delivery ratio comparison chart.
ECGSR has a higher percentage than the CGSR In the ratio of packet delivery.

\subsection{Throughput Analysis}

In the networks the throughput is generally defined as the amount of success data transmission. The following formula is used to calculate the throughput in this context.

Thorughput $=\frac{\text { Total number of packets succesfully transfered }}{\text { Total number of packet transfered }}$ Table.5 Throughput range of protocols

\begin{tabular}{|c|c|c|}
\hline $\begin{array}{c}\text { Number of } \\
\text { nodes }\end{array}$ & CGSR & ECGSR \\
\hline 100 & 330.624 & 292.657 \\
\hline 150 & 380.218 & 340.542 \\
\hline 200 & 280.012 & 230.146 \\
\hline 250 & 620.541 & 583.425 \\
\hline
\end{tabular}

The Table. 5 shows the range of throughput of both protocols CGSR and ECGSR. The ECGSR has the highest throughput compared to the existing protocol CGSR in this analysis of throughput. So, the proposed protocol ECGSR is very efficient than the existing procedure. ECGSR can send the data packet within the least time compared to other protocols with the help of AODV algorithm. Accordingly, it also decreases the packet loss.

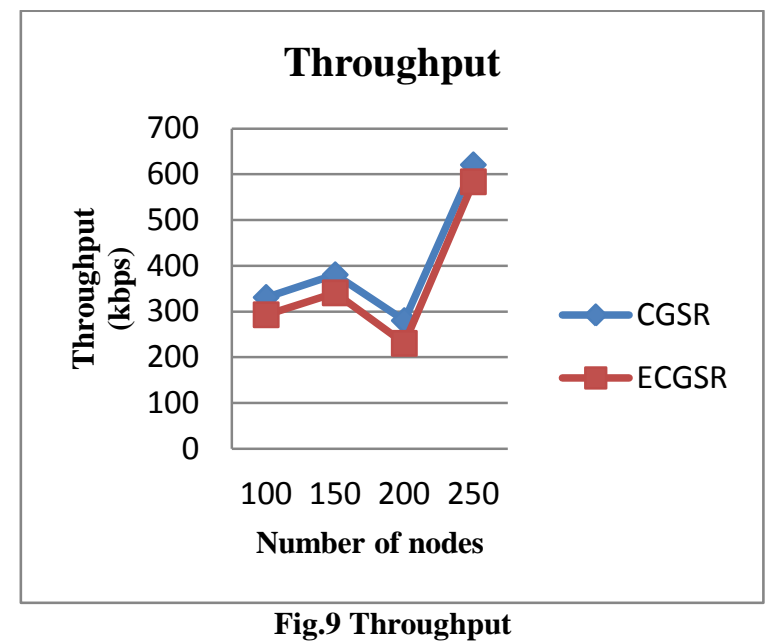

In the simulation analysis of throughput, ECGSR has the highest range of throughput compared to the existing CGSR protocol in Fig.9.

\section{Advantages of ECGSR protocol}

The advantages of ECGSR protocols are

- Reduced end-to-end delay during the data packet transmission.

- Decreased packet drop.

- Improved ratio of packet delivery.

- Increased throughput.

- $\quad$ Reduced routing overhead.

- Extended energy competence and Vifetime.

\section{CONCLUSION}

In this paper discusses about the chosen ECGSR with AODV basis, to reduce the congestion for managing the packet 
delivery ratio, end-to-end delay, overhead and throughput. The clustering is used for grouping the data and finding the Mobile Ad-hoc Networks gateway and cluster heads. The process of ECGSR is to control the congestion for reducing the end-to-end delay, low overhead and packet loss, therefore the energy efficiency and throughput can be achieved by this process. ECGSR can react with the critical congestion in the MANET routing, in which the decisions of routing are created efficiently and can reduce the congestion by controlling the other traffic in the networks. The packet loss, high overhead and end-to-end delay is reduced and energy efficiency, throughput can be increased by this process. In future work, the routing protocols might to be considered as multi-casting hybrid routing protocols.

\section{REFERENCES}

[1] Vinay. P. Viradia, "Improved AODV Routing Protocol for MANET", Journal of Engineering Research and Applications, ISSN: 2248-9622, Vol. 4, Issue 1 (Version 3), pp .368-374, January 2014.

[2] Neelam Phate, Madhvi Saxena and Rizvi M.A, "Minimizing Congestion and Improved QoS of AODV using Clustering in Mobile Ad-hoc Networks", IEEE International Conference on Recent Advances and Innovations in Engineering (ICRAI E-2014), 978-14799-4040-0, May 2014.

[3] Subburam S and Sheik Abdul Khader P, "Predictive congestion control mechanism for MANET", Indian Journal of Computer Science and Engineering, ISSN: 0976-5166 Vol. 3, Issue 5 Oct-Nov 2012.

[4] Heesook Choi, William Enck, Jaesheung Shin, Patrick McDaniel, Thomas F. La Porta, "ASR: Anonymous and Secure Reporting of Traffic Forwarding Activity in Mobile Ad-hoc Networks", National Science Foundation (NSF) grant number CNS-0519460. Volume 15, Issue 4, pp 525-539, May 2009.

[5] Hemant Kumar and Piyush Singh, "TCP Congestion Control with Delay Minimization in MANET", ICICES2014 - S.A.Engineering College, ISBN No.9781-4799-3834-6, 2014

[6] Labiod. H and Quidelleur. A, "QoS-ASR: An Adaptive Source Routing Protocol with QoS support in Multihop Mobile Wireless Networks", 0-7803-7467-3/02, 2002.
[7] Baisakh, "A Review of Energy Efficient Dynamic Source Routing Protocol for Mobile Ad-hoc Networks", International Journal of Computer Applications (0975 8887) Volume 68, Issue 20, April 2013.

[8] Rahim Rashidi and Mohammad Ali Jebraeil Jamali, "Trust Routing Protocol based on Congestion control in MANET”, 978-1-4244-4740-4 IEEE-2009.

[9] Senthilkumaran T and Sankaranarayanan V, "Dynamic congestion detection and control routing in Ad-hoc Networks", Journal of King Saud University- Computer and Information Sciences Vol.25, pp 25-34, 2013.

[10] Govardhan Reddy Kamatam, Podili VS Srinivas and Chandra Sekharaiah K, "Self Congestion Prediction Algorithm for Efficient Routing in Mobile Ad-Hoc Networks", 2014 International Conference on Control, Instrumentation, Communication and Computational Technologies (ICCICCT), 978-1-4799-4190-2, 2014.

[11] Veerlapati Raju, "Dynamic Traffic Congestion Control Scheme in MANET with Cooperative Communication", International Journal for Scientific Research \& Development Vol. 2, Issue 04, ISSN: 2321-0613, 2014.

[12] Ashraf Abu-Ein and Jihad Nader, "An enhanced AODV routing protocol for MANETs", IJCSI International Journal of Computer Science Issues, Vol. 11, Issue 1, No 1, January 2014, ISSN: 1694-0784.

[13] Neetesh Rajpoot, Varsha Sharma, Dr. Jain R. C, "Performance Enhancement of DSR Routing Protocol Using Mobile Agents", International Journal of Engineering and Innovative Technology (IJEIT) Volume 2, Issue 7, January 2013.

[14] Rashmi, Vaibhav Jain and Pawan kumar, " Improved OLSR Protocol in MANET", International Journal of Advanced Research in Computer Science and Software Engineering, Volume 3, Issue 8, August 2013.

[15] Sneha V. Sangolli and Jayavignesh Thyagarajan, "An Efficient Congestion Control Scheme Using CrossLayered Approach and Comparison of TCP Variants for Mobile Ad-hoc Networks (MANETs)", First International Conference on Networks \& Soft Computing, 978-1-4799-3486-7, 2014. 